\title{
ADAPTING QUERY OPTIMIZATION TECHNIQUES FOR EFFICIENT ALERT CORRELATION*
}

\author{
Peng Ning and Dingbang Xu \\ Department of Computer Science \\ North Carolina State University \\ \{pning, dxu\}@ncsu.edu
}

\begin{abstract}
Intrusion alert correlation is the process to identify high-level attack scenarios by reasoning about low-level alerts raised by intrusion detection systems (IDSs). The efficiency of intrusion alert correlation is critical in enabling interactive analysis of intrusion alerts as well as prompt responses to attacks. This paper presents an experimental study aimed at adapting main memory index structures (e.g., T Trees, Linear Hashing) and database query optimization techniques (e.g., nested loop join, sort join) for efficient correlation of intensive alerts. By taking advantage of the characteristics of the alert correlation process, this paper presents three techniques named hyper-alert container, two-level index, and sort correlation. This paper then reports a series of experiments designed to evaluate the effectiveness of these techniques. These experiments demonstrate that (1) hyper-alert containers improve the efficiency of order-preserving index structures (e.g., T Trees), with which an insertion operation involves search, (2) two-level index improves the efficiency of all index structures, (3) a two-level index structure combining Chained Bucket Hashing and Linear Hashing is the most efficient for streamed alerts with and without memory constraint, and (4) sort correlation with heap sort algorithm is the most efficient for alert correlation in batch.
\end{abstract}

Keywords: Intrusion detection, intrusion alert correlation, query optimization

\section{Introduction}

Traditional intrusion detection systems (IDSs) focus on low-level attacks or anomalies, and raise alerts independently, though there may be logical connections between them. In situations where there are intensive intrusions, not only will actual alerts be mixed with false alerts, but the amount of alerts will also

\footnotetext{
*This work is partially supported by the National Science Foundation under grants CCR-0207297 and ITR0219315, by the U.S. Army Research Office under grant DAAD 19-02-1-0219. The authors would like to thank the anonymous reviewers for their valuable suggestions.
} 
become unmanageable. As a result, it is difficult for human users or intrusion response systems to understand the alerts and take appropriate actions.

To assist the analysis of intrusion alerts, several alert correlation methods (e.g., $[6,15])$ have been proposed recently to process the alerts reported by IDSs. As one of the methods, we have developed an alert correlation technique using prerequisites and consequences of attacks [11]. Intuitively, the prerequisite of an intrusion is the necessary condition for the intrusion to be successful, while the consequence of an intrusion is the possible outcome of the intrusion. Based on the prerequisites and consequences of different types of attacks, our method correlates alerts by (partially) matching the consequence of some previous alerts and the prerequisite of some later ones.

We have implemented an offline intrusion alert correlator based on our approach, and the initial experiments indicate that our approach is promising in constructing attack scenarios and differentiating true and false alerts [11]. However, our solution still faces some challenges. In particular, we implemented the previous intrusion alert correlator as a DBMS-based application [11]. Involving a DBMS in the alert correlation process provided enormous convenience and support in intrusion analysis; however, relying entirely on the DBMS also introduced performance penalty. For example, to correlate about 65,000 alerts generated from the DEFCON 8 CTF data set, it took the alert correlator more than 4 minutes. Such performance is clearly insufficient to be practical, especially for interactive analysis of intensive alerts. Our timing analysis indicates that the performance bottleneck lies in the interaction between the intrusion alert correlator and the DBMS. Since this implementation completely relies on the DBMS, processing of each single alert entails interaction with the DBMS, which introduces significant performance penalty.

In this paper, we address this problem by performing alert correlation entirely in main memory, while only using the DBMS as the storage of intrusion alerts. We study several main memory index structures, including Array Binary Search [2], AVL Trees [1], B Trees [3], Chained Bucket Hashing [8], Linear Hashing [10], and T Trees [9], as well as some database query optimization techniques such as nested loop join and sort join [7] to facilitate timely correlation of intrusion alerts. By taking advantage of the characteristics of the alert correlation process, we develop three techniques named hyper-alert container, two-level index, and sort correlation, which further reduce the execution time required by alert correlation.

We performed a series of experiments to evaluate these techniques with the DEF CON 8 CTF data set. The experimental results demonstrate that (1) hyper-alert containers improve the efficiency of index structures with which an insertion operation involves search (e.g., B Trees, T Trees), (2) two-level index improves the efficiency of all index structures, (3) a two-level index structure combining Chained Bucket Hashing and Linear Hashing is most efficient for 
correlating streamed alerts with and without memory constraint, and (4) sort correlation with heap sort algorithm is most efficient for alert correlation in batch. With the most efficient method, the execution time for correlating the alerts generated from the DEF CON 8 CTF data set is reduced from over four minutes to less than one second.

The remainder of this paper is organized as follows. To be self contained, the next section briefly describes our alert correlation method. Section 3 presents our adaptations of the main memory index structures and some join methods. Section 4 reports our implementation and experimental results. Section 5 discusses the related work, and Section 6 concludes this paper and points out some future research directions.

\section{An Overview of Alert Correlation}

This section briefly describes our model for correlating alerts using prerequisites and consequences of intrusions. Further details can be found in [11].

The alert correlation model is based on the observation that in series of attacks, the component attacks are usually not isolated, but related as different stages of the attacks, with the early ones preparing for the later ones. To take advantage of this observation, we correlate alerts using prerequisites and consequences of the corresponding attacks. Intuitively, the prerequisite of an attack is the necessary condition for the attack to be successful, while the consequence of an attack is the possible outcome of the attack if it is successful. We identify the prerequisites (e.g., existence of vulnerable services) and the consequences (e.g., discovery of vulnerable services) of each type of attacks and correlate detected attacks (i.e., alerts) by matching the consequences of previous alerts and the prerequisites of later ones.

We use predicates as basic constructs to represent prerequisites and consequences of attacks. For example, we may use the predicate UDPVulnerableToBOF (VictimIP, VictimPort) to represent the discovery of a vulnerable UDP service. We use a hyper-alert type to encode our knowledge about each type of attacks. A hyper-alert type Tis a triple (fact, prerequisite, consequence) where (1) fact is a set of attribute names, each with an associated domain of values, (2) prerequisite is a logical formula whose free variables are all in fact, and (3) consequence is a set of logical formulas such that all the free variables in consequence are in fact. Intuitively, the fact component of a hyper-alert type gives the information associated with the alert, prerequisite specifies what must be true for the attack to be successful, and consequence describes what could be true if the attack indeed happens. For brevity, we omit the domains associated with attribute names when they are clear from context.

Given a hyper-alert type $T=$ (fact, prerequisite, consequence), a hyper-alert (instance) $h$ of type $T$ is a finite set of tuples on fact, where each tuple is associ- 
ated with an interval-based timestamp [begin_time, end_time]. The hyper-alert $h$ implies that prerequisite must evaluate to True and all the logical formulas in consequence might evaluate to True for each tuple. The fact component of a hyper-alert type is essentially a relation schema (as in relational databases), and a hyper-alert is a relation instance of this schema. A hyper-alert instantiates its prerequisite and consequence by replacing the free variables in prerequisite and consequence with its specific values. Note that prerequisite and consequence can be instantiated multiple times if fact consists of multiple tuples.

To correlate hyper-alerts, we check if an earlier hyper-alert contributes to the prerequisite of a later one. Specifically, we decompose the prerequisite of a hyper-alert into parts of predicates and test whether the consequence of an earlier hyper-alert makes some parts of the prerequisite True (i.e., makes the prerequisite easier to satisfy). If the result is positive, then we correlate the hyper-alerts. In our formal model, given an instance $h$ of the hyper-alert type $T=($ fact , prerequisite, consequence), the prerequisite set (or consequence set, resp.) of $h$, denoted $P(h)$ (or $C(h)$, resp.), is the set of all such predicates that appear in prerequisite (or consequence, resp.) whose arguments are replaced with the corresponding attribute values of each tuple in $h$. Each element in $P(h)$ (or $C(h)$, resp.) is associated with the timestamp of the corresponding tuple in $h$. We say that hyper-alert $h_{1}$ prepares for hyper-alert $h_{2}$ if there exist $p \in P\left(h_{2}\right)$ and $C \subseteq C\left(h_{1}\right)$ such that for all $c \in C$, c.end_time < p.begin_time and the conjunction of all the logical formulas in $C$ implies $p$.

We use a hyper-alert correlation graph to represent a set of correlated hyperalerts. Specifically, a hyper-alert correlation graph $C G=(N, E)$ is a connected graph, where $N$ is a set of hyper-alerts and for each pair $n_{1}, n_{2} \in N$, there is a directed edge from $n_{1}$ to $n_{2}$ in $E$ if and only if $n_{1}$ prepares for $n_{2}$. Figure 1 shows one of the hyper-alert correlation graphs discovered in our experiments with the 2000 DARPA intrusion detection evaluation data sets [11]. Each node in Figure 1 represents a hyper-alert. The numbers inside the nodes are the alert ID's generated by the IDSs.

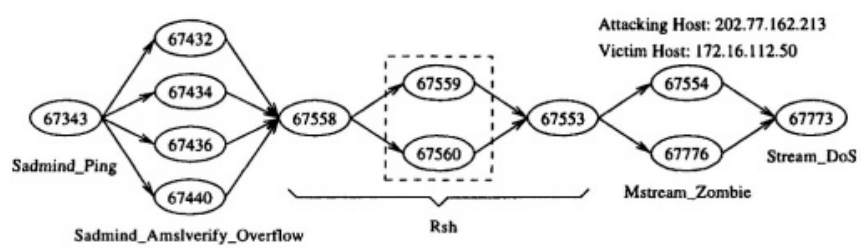

Figure 1. A hyper-alert correlation graph

We have implemented an intrusion alert correlator using our method [11], which is a Java application that interacts with the DBMS via JDBC. In this implementation, we expand the consequence set of each hyper-alert by includ- 
ing all the predicates implied by the consequence set. We call the result the expanded consequence set of the hyper-alert. The predicates in both prerequisite and expanded consequence sets of the hyper-alerts are then encoded into strings called Encoded Predicate and stored in two tables, PrereqSet and ExpandedConseqSet, along with the corresponding hyper-alert ID and timestamp. Both tables have attributes HyperAlertID, EncodedPredicate, begin_time, and end_time, with meanings as indicated by their names. As a result, alert correlation can be performed using the following SQL statement:

\section{SELECT DISTINCT c.HyperAlertID, p.HyperAlertID \\ FROM PrereqSet $p$, ExpandedConseqSet $c$ \\ WHERE $p$.EncodedPredicate $=c$.EncodedPredicate AND c.end_time $<$ p.begin_time}

As discussed earlier, one problem of the intrusion alert correlator is its efficiency because of its dependence on, and intensive interaction with the DBMS. In this paper, we address this problem by performing alert correlation entirely in main memory, while only using the DBMS as the storage of intrusion alerts. In the following, we study how to improve performance of alert correlation by adapting database query optimization techniques, including various main memory index structures.

\section{Adapting Query Optimization Techniques}

The essential problem in this work is how to perform the SQL query in the previous section efficiently. One option is to use database query optimization techniques, which have been studied extensively for both disk based and main memory databases. However, alert correlation has a different access pattern than typical database applications, which may lead to different performance than traditional database applications. In addition, the unique characteristics in alert correlation give us an opportunity for further improvement. Thus, in this section, we seek ways to improve alert correlation by adapting existing query optimization techniques.

In our study, we study the suitability of the following main memory index structures: Array Binary Search [2], AVL Trees [1], B Trees [3], Chained Bucket Hashing [8], Linear Hashing [10], and T Trees [9]. We do not describe them here, since the related information can be found in the references. For comparison purpose, we also implement a naive, sequential scan method, which simply scans in an (unordered) array for the desired data item.

\subsection{Correlating Streamed Intrusion Alerts}

We first study alert correlation methods that deal with intrusion alert streams continuously generated by IDSs. With such methods, an alert correlation system can be pipelined with IDSs and produce correlation result in a timely manner. 
Figure 2 presents a nested loop method that can accommodate streamed alerts. (As the name suggests, nested loop correlation is adapted from nested loop join [7].) It assumes that the input hyper-alerts are ordered ascendingly in terms of their beginning time. The nested loop method takes advantage of main memory index structures such as Linear Hashing. While processing the hyperalerts, it maintains an index structure $\mathcal{I}$ for the instantiated predicates in the expanded consequence sets along with the corresponding hyper-alerts. Each time when a hyper-alert $h$ is processed, the algorithm searches in $\mathcal{I}$ for each instantiated predicate $p$ that appears in $h$ 's prerequisite set. A match of a hyperalert $h^{\prime}$ implies that $h^{\prime}$ has the same instantiated predicate $p$ in its expanded consequent set. If $h^{\prime}$.EndTime is before $h$.BeginTime, then $h^{\prime}$ prepares for $h$. If the method processes all the hyper-alerts in the ascending order of their beginning time, it is easy to see that the nested loop method can find all and only the prepare-for relations between the input hyper-alerts.

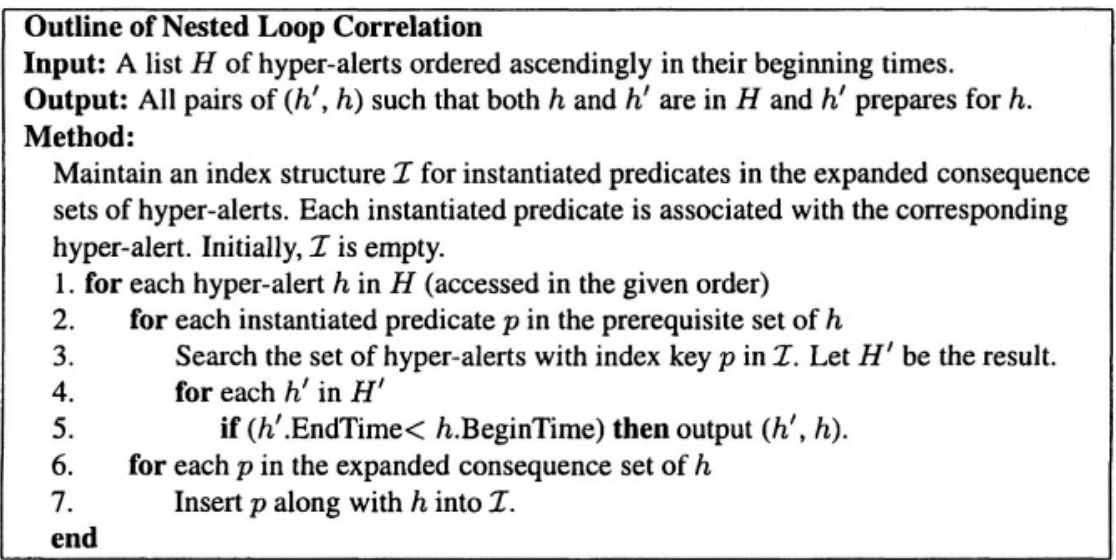

Figure 2. Outline of the nested loop alert correlation methods

The nested loop correlation method has different performance if different index structures are used. Thus, one of our tasks is to identify the index structure most suitable for this method. In addition, we further develop two adaptations to improve the performance of these index structures. Our first adaptation is based on the following observation.

Observation 1 Multiple hyper-alerts may share the same instantiated predicate in their expanded consequence sets. Almost all of them prepare for a later hyper-alert that has the same instantiated predicate in its prerequisite set.

Observation 1 implies that we can associate hyper-alerts with an instantiated predicate $\boldsymbol{p}$ if $\boldsymbol{p}$ appears in the expanded consequence sets of all these hyper-alerts. As a result, locating an instantiated predicate directly leads to the locations of all the hyper-alerts that share the instantiated predicate in their 
expanded consequence sets. We call the set of hyper-alerts associated with an instantiated predicate a hyper-alert container.

Using hyper-alert containers does not always result in better performance. There are two types of accesses to the index structure in the nested loop correlation method: insertion and search. For the index structures that preserve the order of data items, insertion implies search, since each time when an element is inserted into the index structure, it has to be placed in the "right" place. Using hyper-alert container does not increase the insertion cost significantly, while at the same time reduces the search cost. However, for the non-order preserving index structures such as Linear Hashing, insertion does not involve search. Using hyper-alert containers would force to perform a search, since the hyper-alerts have to be put into the right container. In this case, hyper-alert container decreases the search cost but increases the insertion cost, and it is not straightforward to determine whether the overall cost is decreased or not. We study this through experiments later.

Observation 2 There is a small, static, and finite set of predicates. Two instantiated predicates are the same only if they are instantiated from the same predicate.

Observation 2 leads to a two-level index structure. Each instantiated predicate can be split into two parts, the predicate name and the arguments. The top-level index is built on the predicate names. Since we usually have a static and small set of predicate names, we use Chained Bucket Hashing for this purpose. Each element in the top-level index further points to a second-level index structure. The second-level index is built on the arguments of the instantiated predicates. When an instantiated predicate is inserted into a two-level index structure, we first locate the right hash bucket based on the predicate name, then locate the second-level index structure within the hash bucket (by scanning the bucket elements), and finally insert it into the second-level index structure using the arguments.

\subsection{Correlating Intrusion Alerts in Batch}

Some applications allow alerts to be processed in batch (e.g., forensic analysis with an alert database). Though the nested loop method discussed earlier is still applicable, there are more efficient ways for alert correlation in batch.

Figure 3 presents a sort correlation method, which is adapted from sort join [7]. The sort correlation method achieves good performance by taking advantage of efficient main memory sorting algorithms. Specifically, it uses two arrays, $A_{\text {pre }}$ and $A_{\text {con }}$. $A_{\text {pre }}$ stores the instantiated predicates in the prerequisite sets of the hyper-alerts (along with the corresponding hyper-alerts), and $A_{c o n}$ stores the instantiated predicates in the expanded consequence sets (along with 


\section{Outline of Sort Correlation}

Input: A set $H$ of hyper-alerts.

Output: All pairs of $\left(h^{\prime}, h\right)$ such that both $h$ and $h^{\prime}$ are in $H$ and $h^{\prime}$ prepares for $h$.

Method:

Prepare two arrays $A_{\text {pre }}$ and $A_{\text {con }}$, each entry of which is a hyper-alert associated with a $k e y$ field. Each array is initialized with a reasonable size, and reallocated with doubled sizes if out of space. Existing content is copied to the new buffer if reallocation happens.

1. for each $h$ in $H$

2. for each $p$ in the prerequisite set of $h$

3. $\quad$ Append $h$ to $A_{\text {pre }}$ with $k e y=p$.

4. for each $p$ in the expanded consequence set of $h$

5. Append $h$ to $A_{\text {con }}$ with $k e y=p$.

6. Sort $A_{\text {pre }}$ and $A_{\text {con }}$ ascendingly in terms of the key field (with, e.g., heap sort).

7. Partition the entries in $A_{\text {pre }}$ and $A_{\text {con }}$ into maximal blocks that share the same instantiated predicate. Assume $A_{\text {pre }}$ and $A_{\text {con }}$ have $B_{\text {pre }}$ and $B_{\text {con }}$ blocks, resp.

8. $i=0, j=0$.

9. while $\left(i<B_{\text {pre }}\right.$ and $\left.j<B_{\text {con }}\right)$ do

10. if $\left(A_{\text {pre }} . B_{i}\right.$. Predicate $<A_{\text {con }} . B_{j}$. Predicate $)$ then

11. $\quad i=i+1$.

12. else if $\left(A_{\text {pre }} \cdot B_{i}\right.$. Predicate $>A_{\text {con }} . B_{j}$. Predicate $)$ then

13. $j=j+1$.

14. else for each $h$ in $A_{\text {pre }} . B_{i}$ and each $h^{\prime}$ in $A_{\text {con }} . B_{j}$

15. if $h^{\prime}$.EndTime $<$ h.BeginTime then output $\left(h^{\prime}, h\right)$.

16. $i=i+1, j=j+1$.

end

Figure 3. The sort correlation method

the corresponding hyper-alerts). This method then sorts both arrays in terms of the instantiated predicate with an efficient sorting algorithm (e.g., heap sort).

Assume both arrays are sorted ascendingly in terms of instantiated predicate. The sort correlation method partitions both arrays into blocks that share the same instantiated predicate, and scans both arrays simultaneously. It maintains two indices, $i$ and $j$, that references to the current blocks in $A_{\text {pre }}$ and $A_{\text {con }}$, respectively. The method compares the instantiated predicates in the two current blocks. If the instantiated predicate in the current block of $A_{\text {pre }}$ is smaller, it advances the index $i$; if the instantiated predicate in the current block $A_{c o n}$ is smaller, it advances the index $j$; otherwise, the current blocks of $A_{\text {pre }}$ and $A_{\text {con }}$ share the same instantiated predicate. The method then examines each pair of hyper-alerts $h^{\prime}$ and $h$, where $h^{\prime}$ and $h$ are in the current block of $A_{c o n}$ and $A_{p r e}$, respectively. If the end time of $h^{\prime}$ is before the beginning time of $h$, then $h^{\prime}$ prepares for $h$.

It is easy to see that the sort correlation method can find all pairs of hyperalerts such that the first prepares for the second. Consider two hyper-alerts $h$ and $h^{\prime}$ where $h^{\prime}$ prepares for $h$. There must exist an instantiated predicate $p$ in both the expanded consequence set of $h^{\prime}$ and the prerequisite set of $h$. Thus, 
$p$ along with $h^{\prime}$ must be placed in the array $A_{\text {con }}$, and $p$ along with $h$ must be placed in the array $A_{\text {pre. }}$. The scanning method (lines 9-16) will eventually point $i$ to $p$ 's block in $A_{\text {pre }}$ and $j$ to $p$ 's block in $A_{\text {con }}$ at the same time, and output $h^{\prime}$ prepares for $h$. Therefore, the sort correlation can discover all and only pairs of hyper-alerts such that the first prepares for the second.

We also study the possibility of adapting two-index join and hash join methods [7] to improve the performance of batch alert correlation. However, our analysis indicates they cannot outperform nested loop correlation due to the fact that alert correlation is performed entirely in main memory.

A naive adaptation of two-index join leads to the following method: Build two index structures for the instantiated predicates in the prerequisite sets and the expanded consequence sets, respectively. For each instantiated predicate $p$, locate the hyper-alerts related to $p$ in both index structures, and compare the corresponding timestamps. However, this method cannot perform better than the nested loop method. The nested loop method only involves insertion of instantiated predicates in the expanded consequence sets and search of those in the prerequisite sets. In contrast, the above adaptation requires insertion of instantiated predicates in both prerequisite and expanded consequence sets, and search of instantiated predicates in at least one of the index structures.

A possible improvement over the naive adaptation is to merge the two index structures. We can associate two sets of hyper-alerts with each instantiated predicate $p$, denoted $H_{p r e}(p)$ and $H_{c o n}(p)$, and build one index structure for the instantiated predicates. $H_{p r e}(p)$ and $H_{c o n}(p)$ consist of the hyper-alerts that have $\boldsymbol{p}$ in their prerequisite sets and expanded consequence sets, respectively. After all the instantiated predicates in the prerequisite or the consequence set of the hyper-alerts are inserted into the index structure, we can simply scan all the instantiated predicates, and compare the corresponding timestamps of the hyper-alerts in $H_{p r e}(p)$ and $H_{c o n}(p)$ for each instantiated predicate $p$. However, each insertion of an instantiated predicate entails a search operation, since the corresponding hyper-alert has to be inserted into either $H_{p r e}(p)$ or $H_{c o n}(p)$. Thus, this method cannot outperform the nested loop method, which involves one insertion for each instantiated predicate in the expanded consequence sets, and one search for each instantiated predicate in the prerequisite sets. A similar conclusion can be drawn for hash join.

Another possibility to have a faster batch correlation is to use Chained Bucket Hashing. Since the number of alerts is known beforehand, we may be able to decide a relatively accurate hash table size, and thus have a better performance than its counter part for streamed alerts. We study this through experiments later. 


\subsection{Correlating Intrusion Alerts with Limited Memory}

The previous approaches to in-memory alert correlation have assumed that all index structures fit in memory during the alert correlation process. This may be true for analyzing intrusion alerts collected during several days or weeks; however, in typical operational scenarios, the IDSs produce intrusion alerts continuously and the memory of the alert correlation system will eventually be exhausted. A typical solution is to use a "sliding window" to focus on alerts that are close to each other; at any given point in time, only alerts after a previous time point are considered for correlation.

We adopt a sliding window which can accommodate up to $t$ intrusion alerts. The parameter $t$ is determined by the amount of memory available to the intrusion alert correlation system. Since our goal is to optimize the intrusion alert correlation process, we do not discuss how to choose the appropriate value of $t$ in this paper. Each time when a new intrusion alert is coming, we check if inserting this new alert will result in more than $t$ alerts in the index structure. If yes, we remove the oldest alert from the index structure. In either case, we will perform the same correlation process as in Section 3.2. It is also possible to add multiple intrusion alerts in batch. In this case, multiple old alerts may be removed from the index structure. Note that though choosing a sliding time window is another option, it doesn't reflect the memory constraint we have to face in this application.

Using a sliding window in our application essentially implies deleting old intrusion alerts when there are more than $t$ alerts in the memory. This problem appeared to be trivial at the first glance, since all the data structures have known deletion algorithms. However, we soon realized that we had to go through a little trouble to make the deletion efficient. The challenge is that the index structures we build in all the previous approaches are in terms of instantiated predicates to facilitate correlation. However, to remove the oldest intrusion alerts, we need to locate and remove alerts in terms of their timestamps. Thus, the previous index structures cannot be used to perform the deletion operation efficiently. Indeed, each deletion implies a scan of all the alerts in the index structures.

To address this problem, we add a secondary data structure to facilitate locating the oldest intrusion alerts. Since the intrusion alerts are inserted as well as removed in terms of their time order, we use a queue (simulated with a circular buffer) for this purpose. Each newly inserted intrusion alert also has an entry added into this queue, which points to its location in the primary index structure in terms of the instantiated predicates. Thus, when we need to remove the oldest intrusion alert, we can simply dequeue an alert, find its location in the primary index structure, and delete it directly. Indeed, this is more efficient 
than the generic deletion method of the order preserving index structures (e.g., AVL Trees), since deletion usually implies search in those index structures.

\section{Experimental Results}

We have implemented all the techniques discussed in Section 3 in Java, with JDBC to connect to the DBMS. We performed a series of experiments to compare these techniques. All the experiments were run on a DELL Precision Workstation with $1.8 \mathrm{GHz}$ Pentium $4 \mathrm{CPU}$ and $512 \mathrm{M}$ bytes memory. The alerts used in our experiments were generated by a RealSecure Network Sensor 6.0 (http://www. iss . net), which monitors an isolated network in which we replayed the network traffic collected at the DEF CON 8 CTF event. We summarize the results in this section; further details can be found in [12].

Nested-Loop Correlation without Memory Constraint. Our first set of experiments is intended to evaluate the effectiveness of hyper-alert container in nested loop correlation. According to our analysis, hyper-alert container may reduce the execution time if we use the order-preserving index structures. We compared the execution time for Sequential Scan, Array Binary Search, and Linear Hashing, with or without hyper-alert container. Our results show that hyper-alert containers reduce the execution time for Array Binary Search, but increases the execution time for Sequential Scan significantly, and Linear Hashing slightly. Figure 4(a) shows some results obtained in the experiments.

Our second set of experiments is to evaluate the effectiveness of two-level index structure in the nested loop correlation method. Our results indicate that two-level indexes reduce execution time for all index structures. Figure 4(b) shows the improvement of two-level index for B Tree and Linear Hash.

Our next goal is to find out which index structure (with or without the two adaptations) has the best performance for nested loop correlation. Our results show that two-level Linear Hashing (without hyper-alert container) achieves the best performance. Figure 4(c) shows the top three fastest methods, twolevel Array Binary Search with hyper-alert container, two-level AVL Tree, and two-level Linear Hashing.

Batch Correlation without Memory Constraint. Our evaluation here is to determine whether any method can achieve better performance than nested loop correlation with two-level Linear Hashing, the best method for correlating streamed alerts. Besides two-level Linear Hashing, we tested Chained Bucket Hashing and the sort correlation methods because of their potential to outperform two-level Linear Hashing. To further examine the impact of the time order of input hyper-alerts, we examined the timing results with ordered and unordered input (in terms of their timestamps). With unordered input, an algorithm must insert all of the instantiated predicates in the expanded consequence sets before it processes any instantiated predicate in the prerequisite sets. 


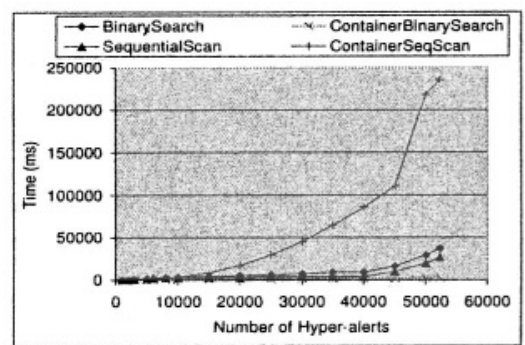

(a) Hyper-alert containers

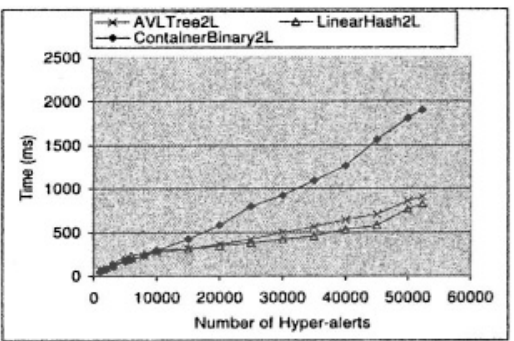

(c) Efficient two-level indices

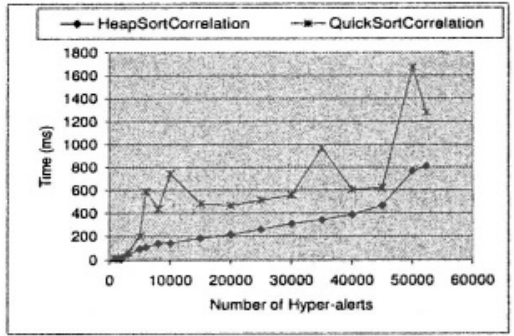

(e) Impact of sorting algorithms

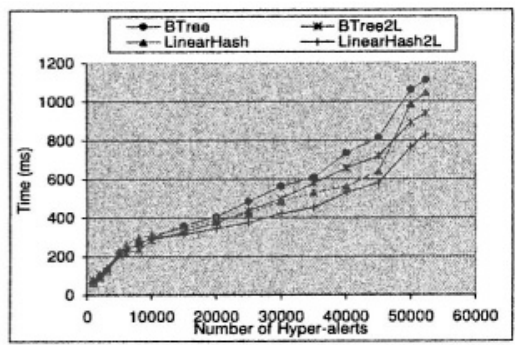

(b) Two-level indices

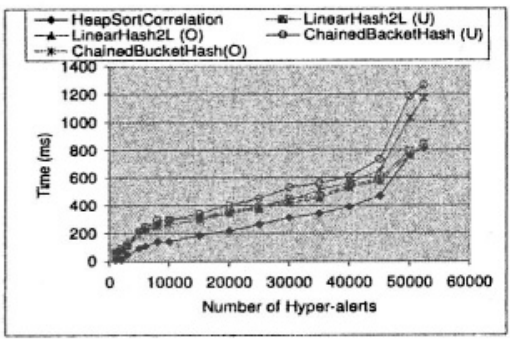

(d) Efficient batch correlation

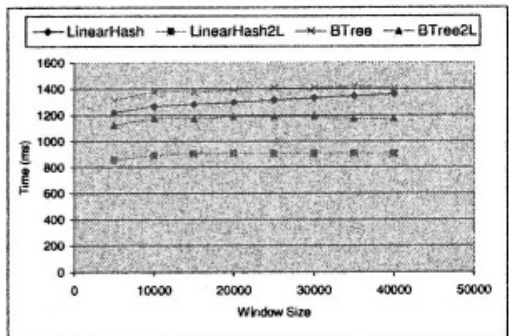

(f) With memory constraint

Figure 4. Experimental results

Figure 4(d) shows the timing results of these methods. Surprisingly, Chained Bucket Hashing has the worst performance. Our further investigation reveals that there is a highly uneven distribution of hyper-alerts in the buckets, which lead to this bad performance. Having ordered input only reduces the execution time slightly for nested loop correlation with both two-level Linear Hashing and Chained Bucket Hashing. Finally, sort correlation with heap sort achieves the best performance among these methods. 
We also study the impact of different sorting algorithms on the execution time of sort correlation. We compare two sorting algorithms, heap sort and quick sort. Heap sort has the least complexity in the worst case scenarios, while quick sort is considered the best practical choice among all the sorting algorithms [4]. Figure 4(e) shows the timing results of both algorithms: Sort correlation with quick sort performs significantly worse (and most unstable) than the heap sort case.

Nested-Loop Correlation with Memory Constraint. Our last set of experiments is focused on evaluating the efficiency of different indexing structures when there is memory constraint. The results indicate that two-level Linear Hashing is the most efficient and the two level index structure improves the performance for all four methods. Figure 4(f) shows some timing results obtained with varying sliding window sizes.

\section{Related Work}

The result in this paper is a continuance of our previous work [11], which has been described earlier. Our method was initially developed to address the limitations of JIGSAW [14]. Our method has several features beyond JIGSAW, including the ability to deal with missing detections and alert aggregation. The work closest to ours is the alert correlation method by Cuppens and Miege [5]. This approach also correlates alerts using partial match of prerequisites (pre-conditions) and consequences (post-conditions) of attacks. However, our method allows alert aggregation during and after correlation, while their approach treats alert aggregation as an individual stage before alert correlation.

Several other alert correlation methods have been proposed. Spice [13] and the probabilistic alert correlation method [15] correlate alerts based on the similarities between alert attributes. Though they are effective in correlating some alerts (e.g., alerts with the same source and destination IP addresses), they cannot fully discover the causal relationships between alerts. Another type of alert correlation methods (e.g., the data mining approach [6]) bases alert correlation on attack scenarios specified by human users or learned through training data sets. These methods are restricted to known attack scenarios. We consider these results complementary to ours.

\section{Conclusions and Future Work}

In this paper, we studied main memory index structures and database query optimization techniques to facilitate timely correlation of intensive alerts. We developed three techniques named hyper-alert container, two-level index, and sort correlation by taking advantage of the characteristics of the alert correlation process. The experimental study demonstrated that (1) hyper-alert containers improve the efficiency of order-preserving index structures, with 
which an insertion operation involves search, (2) two-level index improves the efficiency of all index structures, (3) a two-level index structure combining Chained Bucket Hashing and Linear Hashing is most efficient for streamed alerts with and without memory constraint, and (4) sort correlation with heap sort algorithm is the most efficient for alert correlation in batch. Our future work includes incorporating the efficient methods in this paper into the intrusion alert correlation toolkit and developing more techniques to facilitate timely interactive analysis of intrusion alerts.

\section{References}

[1] A. Aho, J. Hopcroft, and J.D. Ullman. The Design and Analysis of Computer Algorithms. Addison-Wesley, 1974.

[2] A. Ammann, M. Hanrahan, and R. Krishnamurthy. Design of a memory resident DBMS. In Proc. of IEEE COMPCON, February 1985.

[3] D. Comer. The ubiquitous B-Tree. ACM Computeing Surveys, 11(2): 121-137, 1979.

[4] T. H. Cormen, C. E. Leiserson, and R. L. Rivest. Introduction to Algorithms. The MIT Press, 1989.

[5] F. Cuppens and A. Miege. Alert correlation in a cooperative intrusion detection framework. In Proc. of the 2002 IEEE Symposium on Security and Privacy, May 2002.

[6] O. Dain and R.K. Cunningham. Fusing a heterogeneous alert stream into scenarios. In Proc. of the ACM Workshop on Data Mining for Security Applications, November 2001.

[7] H. Garcia-Molina and J. Widom J. D. Ullman. Database System Implementation. Prentice Hall, 2000.

[8] D. Knuth. The Art of Computer Programming. Addison-Wesley, 1973.

[9] T. J. Lehman and M. J. Carey. A study of index structure for main memory database management systems. In Proc. of the 12th Int'l Conf. on Very Large Databases, pages 294-303, August 1986.

[10] W. Litwin. Linear hashing: A new tool for file and table addressing. In Proc. of the 6th Conf. on Very Large Data Bases, pages 212-223, Montreal, Canada, October 1980.

[11] P. Ning, Y. Cui, and D. S Reeves. Constructing attack scenarios through correlation of intrusion alerts. In Proc. of the 9th ACM Conf. on Computer and Communications Security, pages 245-254, November 2002.

[12] P. Ning and D. Xu. Adapting query optimization techniques for efficient intrusion alert correlation. Technical Report TR-2002-13, NCSU Computer Science, August 2002.

[13] S. Stamford, J.A. Hoagland, and J.M. McAlerney. Practical automated detection of stealthy portscans. Journal of Computer Security, 10(1/2):105-136, 2002.

[14] S. Templeton and K. Levitt. A requires/provides model for computer attacks. In Proc. of New Security Paradigms Workshop, pages 31-38, September 2000.

[15] A. Valdes and K. Skinner. Probabilistic alert correlation. In Proc. of the 4th International Symposium on Recent Advances in Intrusion Detection (RAID 2001), pages 54-68, 2001. 